\title{
Combination Strategies of Checkpoint Immunotherapy in Metastatic Breast Cancer
}

This article was published in the following Dove Press journal: OncoTargets and Therapy

\author{
Zhi Bing Liu (D) \\ Luyan Zhang (D) ${ }^{2}$ \\ Jia Bian ${ }^{3}$ \\ Jinbo Jian'
}

'Department of Oncology, Binzhou Medical University Hospital, Binzhou, Shandong Province 256600, People's Republic of China; ${ }^{2}$ Department of Oncology, Binzhou People's Hospital, Binzhou, Shandong Province 256600, People's Republic of China; ${ }^{3}$ Department of Radiology, Binzhou Medical University Hospital, Binzhou, Shandong Province 256600, People's Republic of China
Correspondence: Jinbo Jian Department of Oncology, Binzhou Medical University Hospital, Binzhou, Shandong Province 256600, People's Republic of China

Email jianjinbol23@I26.com

\begin{abstract}
Checkpoint immunotherapy is emerging as a new therapeutic approach for metastatic breast cancer. Monotherapy of immunoagents against PD1/PD-L1 or CTLA-4 has shown little efficacy in these patients. Recently, to determine the optimal use of immunotherapy, there has been a rapid expansion in the number of clinical trials developing immunotherapy combinations. These combination therapeutic approaches can enhance various aspects of cancer immunity, such as tumor antigenicity or intratumor $\mathrm{T}$ cell infiltration, which provides a theoretical basis for combining them with checkpoint immunotherapy to achieve synergistic effects. Here, we review the available data and ongoing efforts to establish the safety and efficacy of immunoagents in combination with chemotherapy, radiotherapy, HER2-targeted therapy, CDK4/6 inhibitors, PARP inhibitors, and another checkpoint immunoagents.
\end{abstract}

Keywords: immunotherapy, breast, cancer, combination

\section{Introduction}

Immune checkpoints are a set of inhibitory pathways that maintain a suppressive level of the immune system. Immunotherapy, which is mainly based on immune checkpoint therapeutic targets, is revolutionizing the treatment of malignancies. ${ }^{1,2}$ Breast cancer remains the most frequent malignancies and causes the second most common cause of cancer death in women. Despite advances in early diagnosis and treatment and a $38 \%$ reduction in breast cancer-related deaths, a large number of patients eventually progress to metastatic disease. ${ }^{3-5}$ There is an urgent need for innovative approaches to treat metastatic breast cancer. Recently, preclinical and clinical data support the key role of immunotherapy in breast cancer. ${ }^{6}$ However, monotherapy of immunoagents is beneficial to less than $10 \%$ of patients with metastatic disease. ${ }^{7}$ Recent research has focused on the development of immunotherapy combinations in determining the optimal use of immunotherapy. In this review, we discuss advances in combined strategies for breast cancer to maximize its clinical effectiveness.

\section{Clinical Progress in Breast Cancer Immunotherapy Anti-PD-I/PD-LI in Breast Cancer}

The PD1 (Programmed Cell Death Protein 1) pathway delivers inhibitory signals that function as a brake for immune response. PD1 acts as an inhibitory immune checkpoint receptor and is expressed in mature T cells, B cells, professional APCs, and natural killer cells. Through interactions with PD-L1 on tumor cells and immune cells, PD1 signaling counters T-cell activation by inhibiting kinases involved in T cell activation. ${ }^{8}$ Also, PD1 engagement inhibits TCR termination signals, and modifies the duration of 
T cells with APCs or target cells, thereby contributing to immune tolerance. ${ }^{9}$ Besides, PD-1 is highly expressed on Treg cells. In the presence of a ligand, PD-1 enhances Treg cell proliferation. ${ }^{9,10}$ Blocking PD-1/PD-L1 signaling can restore immune surveillance and antitumor activity. Multiple agents that inhibit the PD-1/PD-L1 axis has been approved by the US FDA in malignancies. ${ }^{9}$

Pembrolizumab (formerly lambrolizumab, trade name Keytruda) is a humanized antibody used in cancer immunotherapy. Lambrolizumab is a humanized monoclonal IgG4kappa isotype antibody that targets the programmed cell death 1 (PD-1) receptor expressed by $\mathrm{T}$ cells. The antibody is designed to block the negative immunoregulatory signaling of the receptor, thereby activating immune responses to cancer. It has been approved for the treatment in metastatic melanoma. ${ }^{11}$ TNBC (Triple-negative breast cancer, TNBC) is a heterogeneous disease with aggressive tumor pathology. By analyzing gene expression (GE) profiles from 21 breast cancer datasets, Lehmann et al identified 6 TNBC subtypes displaying unique GE and ontologies, including a basal-like (BL1 and BL2), an immunomodulatory (IM), a mesenchymal (M), a mesenchymal stem-like (MSL), and a luminal androgen receptor (LAR) subtype. The IM subtype enriches gene ontologies in immune signaling processes. ${ }^{12,13}$ These processes include immune cell signaling, cytokine signaling, antigen processing and presentation, and signaling through core immune signal transduction pathways. In addition, TNBC generally has a higher expression of PD-L1 compared with other breast cancer subtypes. ${ }^{14}$ The Phase Ib KEYNOTE-012 (NCT02447003) study evaluated the safety and antitumor activity of single-agent pembrolizumab in patients with PDL1-positive metastatic TNBC. Thirty-two patients with PD-L1 -positive TNBC were enrolled and received pembrolizumab $10 \mathrm{mg} / \mathrm{kg}$ intravenously every 2 weeks. Most patients are heavily pretreated. The primary endpoint was overall response rate (ORR). Among the 27 patients evaluable for efficacy, the ORR was $18.5 \%$, including 1 complete response (CR), 4 partial response (PR), and 7 stable disease (SD). The median duration of response (DOR) was not yet reached. Treatment was tolerable. This study provides preliminary evidence of clinical benefits and acceptable safety of pembrolizumab in heavily pretreated metastatic TNBC. ${ }^{15}$ The long-lasting responses were reported with median overall survival (OS) of 10.2 months $(95 \%$ CI, 5.3-17.5) and 12-month OS rate of $41.1 \% .{ }^{16}$ The KEYNOTE-086 (NCT02447003) study investigated the efficacy and safety of single-agent pembrolizumab as a second or later line of treatment in a large group of patients with previously treated metastatic TNBC. 170 patients were enrolled, of which $61.8 \%$ had PD-L1 expression. The primary endpoint was ORR. The median follow-up was 10.9 months. The ORR (95\%) was 5.3\% (2.7-9.9) in the total and 5.7\% (2.4-12.2) in the PD-L1-positive population. DCR was 7.6\% (95\% CI, 4.4-12.7) and 9.5\% (95\% CI, 5.1-16.8), respectively. Median duration of response was not reached in both groups. Median OS was 9 months (95\% CI, 7.7-11.2). Toxicity was manageable. Overall, single-agent pembrolizumab displayed durable efficacy in patients with previously treated metastatic TNBC. ${ }^{17}$

The KEYNOTE-119 (NVT02555657) study is a Phase 3 randomized trial evaluating pembrolizumab monotherapy compared to single-agent chemotherapy of the physician's choice in the second- or third-line treatment of patients with metastatic TNBC. ${ }^{18}$ Outcomes were stratified by PD-L1 tumor status as measured by combined positive score (CPS). The primary endpoint was OS. Overall, 309 patients participated in the pembrolizumab treatment group. 292 patients were enrolled in chemotherapy group. The OS did not reach significant difference between two groups among those with a CPS $\geq 1$ (Hazard ratio $0.86,95 \%$ CI $0.69-1.06$, $\mathrm{p}=0.073$ ) or a CPS $\geq 10$ (Hazard ratio $0.78,95 \%$ CI 0.57 -$1.06, \mathrm{p}=0.057)$. The one hope was an exploratory analysis in patients with a CPS $\geq 20$. Among these patients, the hazard ratio for 12-months OS was 0.58 (95\% CI $0.38-0.88) .{ }^{18}$ Conclusively, the Phase III KEYNOTE-119 study detected a failure in outcomes with the use of pembrolizumab versus chemotherapy for recurrent metastatic TNBC. Nevertheless, these patients with the highest tumor and immune cell level of PD-L1 may benefit from pembrolizumab.

Additionally, the efficacy of single-agent therapy with pembrolizumab was evaluated in $\mathrm{ER}^{+} / \mathrm{HER} 2^{-}$advanced breast cancer (NCT02054806). ${ }^{19}$ This research included 25 cases of $\mathrm{ER}^{+} / \mathrm{HER} 2^{-}$and PD-L1-positive advanced breast cancer. After a median follow-up of 9.7 months, patients treated with pembrolizumab had an ORR of $12.0 \%(95 \%$ CI, 2.5-31.2\%). Three patients experienced PR and none experienced CR. Besides, $16 \%$ of patients experienced stable disease (SD). The median duration of response was 12 months (range, 7.4-15.9 months). In conclusion, for heavily pretreated, PD-L1-positive, ER ${ }^{+} / \mathrm{HER} 2^{-}$advanced breast cancer, pembrolizumab may bring a durable response. A large cohort of studies should be performed in patients to support its utility in ER+/HER2- advanced breast cancer. ${ }^{19}$

Avelumab, a human anti-PD-L1 IgG1 monoclonal antibody, was evaluated in patients with metastatic breast cancer (MBC) form a Phase $1 \mathrm{~b}$ JAVELIN study. ${ }^{20} \mathrm{~A}$ total of 168 heavily pretreated MBC patients (including 58 TNBC 
patients) were treated with avelumab monotherapy. For the total population, the confirmed ORR was $3 \%$, including 1 CR and 4 PRs. For TNBC patients, the ORR was 5.2\%. Besides, a higher ORR was observed in patients with PD-L1 positive versus PD-L1 negative tumor-associated immune cells, both in the overall population ( $16.7 \%$ vs $1.6 \%)$ and in the TNBC group $(22.2 \%$ vs $2.6 \%)$. In conclusion, Avelumab showed tolerability and clinical activity in patients with MBC. High expression of PD-L1 on tumorassociated immune cells may predict response to the treatment of Avelumab. ${ }^{20}$

Atezolizumab is a humanized Ig1 isotype monoclonal antibody that selectively inhibits the binding of PD-L1 to its receptors $\mathrm{PD}-1$ or $\mathrm{B} 71$, thereby reinvigorating anti-tumor immunity of $\mathrm{T}$ cells. ${ }^{21}$ Emens et al investigated the safety and clinical efficacy of single-agent atezolizumab in a metastatic TNBC (mTNBC) cohort. $^{22}$ This phase 1 trial enrolled $116 \mathrm{mTNBC}$ patients regardless of lines of prior therapy. The median follow-up time was 25.3 months (range, 0.4-45.6 months). In first-line patients, the ORR was $24 \%$ (5 of 21) and median OS was 17.6 months (95\% CI, 10.2 months to not estimable). However, in patients receiving atezolizumab as second-line and beyond therapy, ORR was only $6 \%$ (6 of 94) and median OS was 7.3 (95\% CI, 6.1-10.8) months by RECIST. Median DOR of all patients was 21 (range, 3 to $\geq 38$ ) months. Median PFS was 1.4 (95\% CI, 1.3-1.6) months by RECIST. Besides, patients with PD-L1 expression of $\geq 1 \%$ tumor-infiltrating immune cells (ICs) had higher ORR (12\% [11 of 91]) and longer OS (10.1 [95\% CI, 7.0-13.8] months) compared with those with less than $1 \%$ ICs (ORR: 0\% [0 of 21]; OS: 6.0 [95\% CI, 2.6-12.6] months). Atezolizumab was well-tolerated in mTNBC patients. Conclusively, encouraging clinical benefit, especially in first-line individuals or those with higher tumor immune cell infiltration, suggest a promising therapeutic efficacy of atezolizumab in $\mathrm{mTNBC}^{22}$

\section{Anti-CTLA-4 in Breast Cancer}

CTL-associated antigen 4 (CTLA-4) can interact with its target ligand, CD80 or CD86, thereby limiting T-cell activation. $^{23,24}$ Therapeutic blockade of CTLA4 with monoclonal antibodies (mAbs) has been shown to enhance T-cell activation in vivo. Two humanized mAbs targeting CTLA-4, including tremelimumab and ipilimumab, are active in the clinic. $^{25,26}$ Vonderheide et al provided the first evidence for the use of tremelimumab in breast cancer cases. ${ }^{27}$ In this Phase I study, tremelimumab in combination with exemestane was investigated in 26 advanced $\mathrm{ER}+$ and/or $\mathrm{PR}+\mathrm{BC}$ cases. This combination was tolerable in these patients. At the maximum tolerated dose, there were no grade 3 or 4 treatment-related events. The best clinical response was stable disease $\geq 12$ weeks in 11 patients (42\%). In addition, the use of single-agent tremelimumab has not been evaluated in breast cancer patients. ${ }^{27}$ Ipilimumab is an FDA-approved antibody targeting CTLA-4 and has shown well tolerability and activity in metastatic melanoma. In a pilot study, preoperative treatment with ipilimumab alone or in combination with cryoablation was safe and feasible for patients with operable breast cancer without surgical delay. ${ }^{28}$ There have been no trials to establish the clinical feasibility and tolerability of monotherapy of humanized mAbs against CTLA-4 in metastatic breast cancer.

\section{Clinical Progress of Combination Therapy with Immunotherapy in Metastatic Breast Cancer \\ Immune Checkpoint Therapy Plus Radiotherapy}

Radiotherapy has a central role in the treatment of breast cancer. Although historically known to be immunosuppressive, emerging data have recognized that radiation can induce the immune response and inhibit the effect of existing immune checkpoints. The effects of radiotherapy on the immune system are through its contribution to immunogenic cell death, tumor neoantigen presentation, and crosspriming. Radiotherapy can significantly modulate tumor microenvironment and can also increase the percentage of antigen-experienced $\mathrm{T}$ cells and effector memory T cells. ${ }^{29,30}$ In preclinical TNBC models, Dovedi et al showed that low-dose fractionated radiotherapy could upregulate the expression of PD-L1 on tumor cells. ${ }^{31}$ The combination of radiation therapy and PD-1/PD-L1 signaling blockade generated effective antitumor immunity and long-term tumor control. ${ }^{31,32}$ Emerging clinical trials are currently ongoing to investigate the use of combined immunoradiotherapy in the treatment of breast cancer. A phase 1 pilot study (NCT02303366) aimed to describe the safety and biological effects of combining stereotactic ablative body radiosurgery (SABR) and pembrolizumab in patients with oligometastatic breast carcinoma. The study was completed normally, and we look forward to the final results. Another single-arm Phase II study (NCT02730130) evaluated the efficacy of pembrolizumab plus radiotherapy in patients with metastatic TNBC. 17 patients were enrolled. The median number of prior chemotherapy lines was 3 . Pembrolizumab 200mg was administrated intravenously 
within 3 days of the first radiation fraction. The primary endpoint was overall survival at week 13 . Finally, 9 patients were evaluated for efficacy at week 13. Three of them (33\%) had a partial response. One patient (11\%) had stable disease. 5 cases progressed after treatment. Both partial and stable responses were durable for at least 21 weeks. The combination was well-tolerated. ${ }^{33}$ Besides, a phase II trial (NCT02499367) from the Netherlands aims to investigate if short-term induction treatment with radiation could contribute to synergistic activity with nivolumab in TNBC patients. Its current status is recruiting.

In a poorly immunogenic metastatic mouse breast cancer model, treatment with local radiation and CTLA-4 blockade could induce significant anti-tumor immunity and prolong the survival of mice. ${ }^{34}$ Jiang et al evaluated the combination of local radiation and tremelimumab in patients with inoperable locally recurrent or metastatic breast cancer. The primary objective was to investigate the maximum tolerated dose (MTD) of combination therapy. The second objective was to evaluate clinical response. This trial has a great limitation that only 6 patients were recruited. One stable disease was achieved as the best response. Tremelimumab at $3 \mathrm{mg} / \mathrm{kg}$ combined with radiation therapy appears to be well-tolerated. Due to the extremely small sample size, it is difficult to discern any definitive conclusion from this trial. ${ }^{35}$

\section{Combination Therapy of Immunotherapy and Chemotherapy}

The combination of chemotherapy may be synergistic in the process of immunotherapy. The rationale is that chemotherapy may induce the concomitant release of tumor antigens, a decrease of inhibitory immune cell populations, and an increase of cytokine mediators. ${ }^{36} \mathrm{Nab}$-paclitaxel is a rational partner and is thought to affect the activity of immunotherapy. A phase 1b clinical trial (NCT01633970) investigated the safety, tolerability and clinical activity of atezolizumab combined with nab-paclitaxel in metastatic TNBC. Thirty-three women with stage IV or locally recurrent TNBC were enrolled. The median follow-up time was 24.4 months. Patients were given concurrent intravenous atezolizumab and intravenous nab-paclitaxel. The primary endpoints were safety and tolerability. All patients $(100 \%)$ had at least 1 treatmentrelated adverse event. About 24 patients (73\%) had grade 3/4 adverse events. The toxicity of dual therapy was manageable. The ORR was 39.4\% (95\% CI, 22.9-57.9\%), including $1 \mathrm{CR}$ and 12 PRs. The DCR was 51.5\% (95\% CI, 33.5-69.2\%). Conclusively, they detected a manageable toxicity profile and clinical efficacy of combination therapy in mTNBC. Large randomized clinical trials are needed to confirm its efficacy. ${ }^{37}$

IMpassion130 (NCT02425891) was the first randomized Phase III trial to demonstrate the efficacy of atezolizumab plus nab-paclitaxel in untreated metastatic TNBC. A total of 902 patients were enrolled from 41 countries, of which $38.6 \%$ from Europe, 25.5\% from United States and Canada, 16.1\% from Asia, $15.2 \%$ from Latin America and 4.7\% from Australia. 902 patients were randomly assigned at a ratio of 1:1 to receive atezolizumab plus nab-paclitaxel or place plus nab-paclitaxel. Prior to treating patients with atezolizumab, tumor samples were evaluated for PD-L1 expression by immunohistochemical testing. PD-L1 expression was assessed in tumor-infiltrating immune cells as a percentage of tumor area $(<1 \%$ [ $=$ PD-L1 negative] or $\geq 1 \%$ [ $=$ PD-L1 positive]). The primary endpoints was PFS and OS. The median PFS were 7.2 months in the combination group and 5.5 months in the monotherapy group (HR, 0.8; 95\% CI, 0.69-0.92; $\mathrm{p}=0.002$ ). Among patients displaying PD-L1-positivity, the median PFS was 7.5 months and 5.0 months, respectively (HR, 0.62; 95\% CI, 0.49-0.78; $\mathrm{p}<0.001$ ). In the interim OS analysis, the difference in OS did not reach significance in the intention-to-treat (ITT) population. Due to the hierarchical statistical analysis procedure, there was no formal testing of OS in the PD-L1-positive population. However, Kaplan-Meier analyses showed a meaningful improvement in the PD-L1positive group: the OS hazard ratio was 0.62 (95\% CI:0.45-0.86), and the median OS was 25.0 months in the atezolizumab-nab-paclitaxel group compared to 15.5 months in the placebo-nab-paclitaxel group. Recently, an updated OS analysis was reported at ASCO 2019. ${ }^{38}$ The updated interim OS analysis was consistent with the 1st analysis. The median OS data of ITT patients did not reach statistical significance, but a 7.0-month improvement in median OS was observed in PD-L1-positive patients with combination treatment compared with atezolizumab monotherapy. The combination regimen was tolerated and consistent with the known safety profiles of both drugs. ${ }^{39}$ These results led to accelerated FDA approval of atezolizumab in combination with nabpaclitaxel for patients with PD-L1-positive metastatic TNBC.

Eribulin mesylate in combination with pembrolizumab has been also investigated in patients with metastatic TNBC. ${ }^{40}$ The updated results of ENHANCE1 (NCT02513472) showed that the combination of eribulin and pembrolizumab resulted in an ORR of $26.4 \%$ (95\% CI, 18.3-35.9). Treatmentemergent adverse events (TEAEs) for the combination group were similar to those observed in each therapy. Eribulin combined with pembrolizumab has shown efficacy in patients with 
mTNBC, regardless of PD-L1 expression or prior lines of chemotherapy. Of the 106 evaluable mTNBC patients, 49 were PD-L1-positive and the ORR was $30.6 \%$. For patients with PD-L1-negative ( $n=49)$, the ORR was $22.4 \%$. The results of ENHANCE 1 provide us with hope for studying the combination of eribulin and checkpoint inhibitors in the treatment of metastatic breast cancer. Doxorubicin and cyclophosphamide have been reported to induce immunogenic cell death and type 1 interferon immune responses in breast cancer. An ongoing trial (NCT03409198) is ongoing to evaluate Doxorubicin and cyclophosphamide combined with Ipilimumab and Nivolumab in patients with metastatic hormone receptor-positive breast cancer. Another ongoing phase II trial (NCT03095352) is underway to investigate pembrolizumab plus carboplatin compared to carboplatin alone in breast cancer with chest wall metastasis.

\section{Combination Therapy of Immunotherapy and HER-2-Targeted Therapy}

Preclinical studies have demonstrated that the combination of HER-2 and PD-L1 inhibitors has a synergistic effect in HER-2-positive breast cancer. ${ }^{41}$ Besides, Tregs were found to be increased in HER-2-positive breast cancer patients who failed to achieve pCR, which displays the development of an immunosuppressive phenotype. ${ }^{42}$ Trastuzumab has been reported to modulate immune activity, mediate antibody-dependent cellular cytotoxicity (ADCC) and promote T-cell response. ${ }^{43}$ Clinically, data from the PANACEA study (NCT02129556) demonstrated the durable clinical benefit of pembrolizumab plus trastuzumab in patients with PD-L1-positive, trastuzumab-resistant, advanced, HER-2-positive breast cancer. In this single-arm, multicenter trial, 6 patients were enrolled in phase $1 \mathrm{~b}$ and 52 patients in Phase 2. For the phase $1 \mathrm{~b}$ trial, no dose-limiting toxicities were tested. In the phase 2 trial, six of the forty PD-L1positive patients achieved an objective response when treated with the flat dose of pembrolizumab plus standard trastuzumab. However, in PD-L1-negative tumors, there were no objective responders. Pembrolizumab combined with trastuzumab was well tolerated and had durable antitumor activity in patients with PD-L1-positive tumors. ${ }^{44}$ Besides, the use of pembrolizumab in combination with carboplatin and trastuzumab for advanced HER-2-positive breast cancer is being evaluated in a randomized phase II trial (NCT03095352).

The KATE2 trial was the first study to study atezolizumab combined with trastuzumab emtansine (T-DM1) in previously treated HER-2-positive metastatic breast cancer
(NCT02924883). Of the 202 patients who progressed after treatment with trastuzumab and a taxane, they were randomly assigned in a 2:1 manner to receive atezolizumab or placebo plus TDM1. The primary endpoint was investigatorassessed PFS. The KATE2 trial revealed no statistically significant difference in PFS among ITT population who received atezolizumab plus TDM1 (PFS was 8.2 months; 95\% CI 5.8-10.7) relative to placebo plus TDM1 (PFS: 6.8 months, 95\% CI 4.0-11.1) (HR was 0.82; 95\% CI 0.55-1.23; $\mathrm{p}=0.3332$ ). The combination group did not demonstrate a clinically significant PFS benefit when compared with TDM1 monotherapy. ${ }^{45}$ At the 2019 ESMO Congress, the final analysis of KATE2 for efficacy and safety was updated. In the ITT population, 1 -year OS was $89 \%$ in both groups. In the PD-L1 immune cell-positive subgroup, the 1-year OS data was numerically higher in the atezolizumab plus TDM1 arm than in the placebo plus TDM1 arm (1-year survival rate of $94 \%$ vs $88 \%$; HR $=0.74,95 \%$ CI: $0.42-1.30) .{ }^{46}$ Grade $3-5$ adverse events occurred in $52.6 \%$ of those who received atezolizumab versus $44.8 \%$ who received placebo. Further, atezolizumab in combination with T-DM1 or with trastuzumab and pertuzumab are also being evaluated in patients with HER-2-positive breast cancer by a phase 1b, open-label study (NCT02605915). A single-arm, phase 2a clinical trial investigated the safety and efficacy of combining atezolizumab with paclitaxel, trastuzumab, and pertuzumab in patients with locally advanced or metastatic HER-2-positive breast cancer (NCT03125928).

A phase 1 study assessed the safety and clinical activity of durvalumab and trastuzumab in heavily pretreated HER-2-positive MBC patients. Patients were enrolled on a standard $3+3$ dose escalation schedule. 14 patients who had $<1 \%$ PD-L1 expression were evaluable for the response. There was no dose-limiting toxicities at dose level $1(n=6)$ or dose expansion $(n=9)$ during cycle 1 . No responses were observed, only with $29 \%$ patients showing stable disease at week 6 . In this phase 1 trial, no significant efficacy was detected in heavily pre-treated HER-2 positive MBC patients. ${ }^{47}$

\section{Combination Therapy of Immune Checkpoint Therapy and PARP Inhibitors}

PARP inhibitors could attenuate anticancer immunity via upregulating the level of PD-L1 in breast cancer cell lines and animal models. Inhibition of PD-L1 signaling resensitized PARP-treated cells to T-cell killing. ${ }^{48}$ The combination of PARP inhibition and PD-L1 blockade significantly delayed tumor outgrowth in vivo. The MEDIOLA trial 
(NCT02734004) evaluated whether the combination of olaparib and durvalumab could lead to clinical activity in BRCA1/2-mutated and HER-2-negative MBC. Eligible patients were not allowed to receive PARP inhibitors or immunotherapy, and had previous received no more than two lines of chemotherapy. Patients were administrated with $300 \mathrm{mg}$ bid olaparib daily for 4 weeks, followed by $1.5 \mathrm{~g}$ intravenous durvalumab once every 4 weeks in addition to olaparib. The dual primary endpoints were disease control rate (DCR) at 12 weeks and safety. 34 patients were analyzed for safety, and 30 patients for efficacy analysis. The DCR at 12 weeks was 24/30 (80\%). The DCR at 28 weeks was 15/30 (50\%). 19 confirmed responses were observed. This combination was welltolerated with safety consistent with the individual agent profiles. ${ }^{49}$ Conclusively, the combination of PARP inhibition and immune checkpoint therapy showed preliminary clinical activity in BRCA1/2-mutated HER-2-negative MBC.

The TOPACIO/Keynote-162 study (NCT02657889) evaluated the safety and efficacy of a combination therapy regimen with niraparib + pembrolizumab in patients with mTNBC. Patients were administrated with $200 \mathrm{mg}$ of oral niraparib once daily and $200 \mathrm{mg}$ of intravenous pembrolizumab on day 1 of each 21-day cycle. The primary endpoint was ORR. Recently, the preliminary results were reported. Forty-seven patients were evaluable for efficacy and treated with a median of 1 prior line of therapy in the metastatic setting. The ORR was $21 \%$, including $5 \mathrm{CRs}$, 5 confirmed PRs, 13 SDs. Besides, of the 15 evaluable patients with BRCA mutations, 7 had objective responses (ORR was 47\%; 90\% CI, 24-70\%), and median PFS was 8.3 months (95\% CI, 2.1 months to not estimable). Among the 27 patients with tumor BRCA wild-type status, the ORR included $3(11 \% ; 90 \%$ CI, 3-26\%), and median PFS was 2.1 months (95\% CI, 1.4-2.5 months). The patients with germline BRCA1/2 mutations had higher ORR than patients with wild-type tumors. Patients with PD-L1-positive disease had better responses than those with PD-L1-negative cancers. Preliminary efficacy is encouraging with durable responses in patients with mTNBC. ${ }^{50,51}$ However, the previous trials have obvious limitations with the lack of a comparator arm. Therefore, we could not draw strong conclusions on the role of combination therapy in mTNBC management. The findings presented here need to be validated in a larger clinical trial.
Combination Therapy of Immune Checkpoint Therapy and Cyclin-Dependent Kinases 4/6 (CDK4/6) Inhibitors Pharmacological inhibitors of CDK4/6 have shown significant activity in patients with ER-positive, HER2-negative metastatic breast cancer. ${ }^{52-54}$ Inhibition of CDK4/6 works primarily by suppressing retinoblastoma phosphorylation and inducing G1 cell cycle arrest in cancer cells. CDK4/6 inhibitors have been also proven to enhance anti-tumor immune response. It can stimulate the production of type III interferons, thereby increasing tumor antigen presentation. Besides, CDK4/6 inhibition could significantly inhibit the proliferation of immunosuppressive regulatory T cells. ${ }^{55}$ These findings indicate that there may be a clinically effective synergy between CDK4/6 inhibitors and immunotherapy. This has been validated in murine syngeneic tumor models. As a selective CDK4/6 inhibitor, monotherapy of Abemaciclib resulted in delayed tumor growth, which was associated with an increased $\mathrm{T}$ cell inflammatory signature in tumors. $^{56}$ A phase I study (JPBJ, NCT02079636) investigated abemaciclib plus pembrolizumab in patients with $\mathrm{HR}+$, HER2- MBC. The primary endpoint was to study the safety profile of combination therapy. Twenty-eight patients were enrolled. Four patients (14\%) showed an objective response at 24 weeks. This response was higher than the response (11\%) observed in patients treated with abemaciclib monotherapy at corresponding early time points in the MONARCH 1 study. Besides, the combination group demonstrated a generally manageable profile. ${ }^{57}$

\section{Dual Checkpoint Blockade Therapy}

In melanoma, concurrent therapy with anti-PD-1 (nivolumab) and anti-CTLA-4 (ipilimumab) had a manageable safety profile. The deep and durable clinical tumor responses were observed in patients treated with the combination therapy comparing favorably to monotherapy with ipilimumab or nivolumab alone. ${ }^{58,59}$ This suggests that combination therapy could "rescue" participants who were less likely to respond to a single anti-PD1/PD-L1 agent. These data have inspired clinical trials of dual checkpoint blockade therapy in metastatic breast cancer, but only a small pilot study has reported outcomes to date. This single-arm pilot study was designed to determine the ORR of anti-PD-L1 (durvalumab) and antiCTLA-4 (tremelimumab) in metastatic ER-positive BC and TNBC. $^{60} 18$ patients were accrued (11 ER-positive breast cancer; 7 TNBC). 3 responses were achieved and only observed in patients with TNBC. Conclusively, in this small pilot study, combination durvalumab and tremelimumab have low response rates in unselected $\mathrm{MBC}$; however, 
Table I Trials Evaluating the Use of Immune Checkpoint Inhibitor in Combination with Radiotherapy, Molecular Therapies or Chemotherapy That Reported the Preliminary Results in Metastatic Breast Cancer

\begin{tabular}{|c|c|c|c|c|c|c|}
\hline $\begin{array}{l}\text { NCT } \\
\text { Number }\end{array}$ & $\begin{array}{l}\text { Immune } \\
\text { Checkpoint } \\
\text { Inhibitor }\end{array}$ & $\begin{array}{l}\text { Combination } \\
\text { Therapy }\end{array}$ & Phase & $\begin{array}{l}\text { Number } \\
\text { of } \\
\text { Enrolled } \\
\text { Patients }\end{array}$ & $\begin{array}{l}\text { Enrolled } \\
\text { Patients }\end{array}$ & Key Results \\
\hline \multicolumn{7}{|l|}{ Radiotherapy } \\
\hline NCT02730I30 & Pembrolizumab & Radiotherapy & Phase I & 17 & $\begin{array}{l}\text { Metastatic } \\
\text { TNBC }\end{array}$ & $\begin{array}{l}\text { Three of nine evaluable patients (33\%) had PRs. } \\
\text { One patient (II\%) had SD. }\end{array}$ \\
\hline- & Tremelimumab & Local radiation & Phase I & 6 & $\begin{array}{l}\text { Locally } \\
\text { recurrent or } \\
\text { metastatic BC }\end{array}$ & $\begin{array}{l}\text { One SD was achieved as the best response. } \\
\text { Tremelimumab at } 3 \mathrm{mg} / \mathrm{kg} \text { combined with radiation } \\
\text { therapy was well-tolerated. }\end{array}$ \\
\hline \multicolumn{7}{|c|}{ Chemotherapy } \\
\hline NCT0I633970 & Atezolizumab & Nab-paclitaxel & Phase Ib & 33 & $\begin{array}{l}\text { Metastatic } \\
\text { TNBC }\end{array}$ & $\begin{array}{l}\text { The ORR was } 39.4 \%(95 \% \mathrm{Cl}, 22.9-57.9 \%) \text {, including I } \\
\text { CR and I2 PRs. } \\
\text { The DCR was } 51.5 \%(95 \% \mathrm{Cl}, 33.5-69.2 \%) \text {. }\end{array}$ \\
\hline NCT0242589I & Atezolizumab & Nab-paclitaxel & Phase 3 & 902 & $\begin{array}{l}\text { Untreated } \\
\text { metastatic } \\
\text { TNBC }\end{array}$ & $\begin{array}{l}\text { Median PFS were } 7.2 \text { months }(95 \% \mathrm{Cl} 5.6-7.5) \text { and } \\
\text { median OS was } 21.3 \text { months }(95 \% \mathrm{Cl} 17.3-23.4) \text { for all } \\
\text { patients. } \\
\text { Median OS for PD-LI+ patients was } 25.0 \text { months }(95 \% \\
\mathrm{Cl} 22.6 \text {-not established) }\end{array}$ \\
\hline NCT025I3472 & Pembrolizumab & $\begin{array}{l}\text { Eribulin } \\
\text { mesylate }\end{array}$ & Phase $1 / 2$ & 106 & $\begin{array}{l}\text { Metastatic } \\
\text { TNBC }\end{array}$ & $\begin{array}{l}\text { For the } 49 \mathrm{PD}-\mathrm{LI} \text {-positive patients, the ORR was } \\
30.6 \% \text {. } \\
\text { For patients with PD-LI-negative ( } n=49 \text { ), the ORR was } \\
22.4 \% \text {. }\end{array}$ \\
\hline \multicolumn{7}{|c|}{ HER-2-targeted therapy } \\
\hline NCT02I 29556 & Pembrolizumab & Trastuzumab & Phase Ib/2 & $\begin{array}{l}\text { Phaselb:6 } \\
\text { phase 2:52 }\end{array}$ & $\begin{array}{l}\text { PD-LI+, } \\
\text { trastuzumab-R, } \\
\text { advanced, } \\
\text { HER2+BC }\end{array}$ & $\begin{array}{l}\text { Phase Ib trial, there is no dose-limiting toxicities. } \\
\text { Phase } 2 \text { trial, six ( } 15 \%, 90 \% \mathrm{Cl} 7-29) \text { of } 40 \mathrm{PD}-\mathrm{LI}+ \\
\text { patients achieved an OR. There were no objective } \\
\text { responders among the PD-LI- patients. }\end{array}$ \\
\hline NCT02924883 & Atezolizumab & T-DMI & Phase 2 & 202 & $\begin{array}{l}\text { Previously } \\
\text { treated HER2+ } \\
\text { MBC }\end{array}$ & $\begin{array}{l}\text { Among ITT population: no statistically significant } \\
\text { difference in PFS between combination therapy and single- } \\
\text { agent TDMI. } \\
\text { In the ITT population, I-year OS was } 89 \% \text { in both groups. } \\
\text { In the PD-LI+ subgroup, I-year survival rate was } 94 \% \text { for } \\
\text { combination group vs } 88 \% \text { for TDMI. }\end{array}$ \\
\hline NCT02649686 & Durvalumab & Trastuzumab & Phase I & 14 & $\begin{array}{l}\text { Heavily } \\
\text { pretreated } \\
\text { HER2+ MBC }\end{array}$ & $\begin{array}{l}\text { No responses were observed, only with } 29 \% \text { patients } \\
\text { showing stable disease at week } 6 \text {. }\end{array}$ \\
\hline \multicolumn{7}{|c|}{ PARP inhibitors } \\
\hline NCT02734004 & Durvalumab & Olaparib & Phase I/2 & 34 & $\begin{array}{l}\text { BRCAI/ } \\
\text { 2-mutated and } \\
\text { HER2- MBC }\end{array}$ & $\begin{array}{l}\text { The DCR at } 12 \text { weeks was } 24 / 30(80 \%) \text {. } \\
\text { The DCR at } 28 \text { weeks was } 15 / 30(50 \%) \text {. } \\
19 \text { confirmed responses were observed. } \\
\text { This combination was well-tolerated with safety. }\end{array}$ \\
\hline NCT02657889 & Pembrolizumab & Niraparib & Phase 2 & 47 & mTNBC & $\begin{array}{l}\text { The ORR was } 21 \% \text {, including } 5 \text { CRs, } 5 \text { confirmed PRs, } \\
13 \text { SDs. }\end{array}$ \\
\hline
\end{tabular}

(Continued) 
Table I (Continued).

\begin{tabular}{|c|c|c|c|c|c|c|}
\hline $\begin{array}{l}\text { NCT } \\
\text { Number }\end{array}$ & $\begin{array}{l}\text { Immune } \\
\text { Checkpoint } \\
\text { Inhibitor }\end{array}$ & $\begin{array}{l}\text { Combination } \\
\text { Therapy }\end{array}$ & Phase & $\begin{array}{l}\text { Number } \\
\text { of } \\
\text { Enrolled } \\
\text { Patients }\end{array}$ & $\begin{array}{l}\text { Enrolled } \\
\text { Patients }\end{array}$ & Key Results \\
\hline \multicolumn{7}{|c|}{ CDK4/6 inhibitors } \\
\hline NCT02079636 & Pembrolizumab & Abemaciclib & Phase I & 28 & $\begin{array}{l}\text { ER+, HER2- } \\
M B C\end{array}$ & $\begin{array}{l}\text { Four patients (14\%) showed an objective response at } \\
24 \text { weeks. }\end{array}$ \\
\hline \multicolumn{7}{|c|}{ Dual checkpoint blockade therapy } \\
\hline NCT02536794 & Durvalumab & Tremelimumab & Phase 2 & 18 & $\begin{array}{l}\text { Metastatic ER } \\
+\mathrm{BC} \text { and } \\
\text { TNBC }\end{array}$ & $\begin{array}{l}3 \text { responses were achieved and only observed in } \\
\text { patients with TNBC }\end{array}$ \\
\hline
\end{tabular}

Abbreviations: NCT, clinicalTrials.gov registry number; Cl, confidence interval; Nab-paclitaxel, nanoparticle albumin-bound paclitaxel; PARP, poly (ADP-ribose) polymerase; T-DMI, trastuzumab emtansine; trastuzumab-R, trastuzumab-resistant; PD-LI, programmed death ligand-I; ER, estrogen receptor; HER2, human epidermal growth factor receptor 2; TNBC, triple-negative breast cancer; mTNBC, metastatic triple-negative breast cancer; MBC, metastatic breast cancer; BC, breast cancer; DCR, disease control rate; ORR, overall response rate; OR, objective response; CR, complete response; PR, partial response; SD, stable disease; PFS, progression-free survival; OS, overall survival; ITT, Intention to treat; BRCA, breast cancer gene.

higher rates of clinical benefit were observed in TNBC. Further research is warranted in TNBC to confirm the findings.

\section{Conclusion}

Over the past decades, checkpoint blockade strategies are evolving dramatically in the treatment of melanoma and numerous solid malignancies. Checkpoint immunotherapy has emerged as a promising treatment for breast cancer, especially for TNBC. However, monotherapy of immunoagents has a modest benefit in patients with metastatic disease. There is a growing interest in testing clinical activity and tolerability for incorporating cytotoxic agents, radiation, targeted therapies, or dual checkpoint blockade (Table 1). Despite the accumulating evidence supporting combination therapies, finding the best combination for individual patients will require to address the problems of developing predictive biomarkers and crucial selection of breast cancer patients who would benefit most from immunotherapy combinations. In general, an exciting new area of checkpoint immunotherapy is developing. The results of ongoing trials will be generated in the next few years. We hope to see these efforts translated into clinical benefits.

\section{Disclosure}

The authors have declared that no competing interest exists in this work.

\section{References}

1. Emens LA. Breast cancer immunobiology driving immunotherapy: vaccines and immune checkpoint blockade. Expert Rev Anticancer Ther. 2012;12(12):1597-1611. doi:10.1586/era.12.147

2. Vonderheide RH, Domchek SM, Clark AS. Immunotherapy for breast cancer: what are we missing? Clin Cancer Res. 2017;23 (11):2640-2646. doi:10.1158/1078-0432.CCR-16-2569

3. Jones SE. Metastatic breast cancer: the treatment challenge. Clin Breast Cancer. 2008;8(3):224-233. doi:10.3816/CBC.2008.n.025

4. Siegel RL, Miller KD, Jemal A. Cancer statistics, 2017. CA Cancer J Clin. 2017;67(1):7-30. doi:10.3322/caac.21387

5. Key TJ, Verkasalo PK, Banks E. Epidemiology of breast cancer. Lancet Oncol. 2001;2(3):133-140. doi:10.1016/S1470-2045(00)00254-0

6. Lipson EJ, Forde PM, Hammers HJ, Emens LA, Taube JM, Topalian SL. Antagonists of PD-1 and PD-L1 in cancer treatment. Semin Oncol. 2015;42(4):587-600. doi:10.1053/j.seminoncol.2015.05.013

7. Emens LA. Breast cancer immunotherapy: facts and hopes. Clin Cancer Res. 2018;24(3):511-520. doi:10.1158/1078-0432.CCR-16-3001

8. Pedoeem A, Azoulay-Alfaguter I, Strazza M, Silverman GJ, Mor A. Programmed death-1 pathway in cancer and autoimmunity. Clin Immunol. 2014;153(1):145-152. doi:10.1016/j.clim.2014.04.010

9. Pardoll DM. The blockade of immune checkpoints in cancer immunotherapy. Nat Rev Cancer. 2012;12(4):252-264. doi:10.1038/ $\operatorname{nrc} 3239$

10. Francisco LM, Salinas VH, Brown KE, et al. PD-L1 regulates the development, maintenance, and function of induced regulatory $\mathrm{T}$ cells. J Exp Med. 2009;206(13):3015-3029. doi:10.1084/jem.20090847

11. Poole RM. Pembrolizumab: first global approval. Drugs. 2014;74 (16):1973-1981. doi:10.1007/s40265-014-0314-5

12. Lehmann BD, Bauer JA, Chen X, et al. Identification of human triple-negative breast cancer subtypes and preclinical models for selection of targeted therapies. $J$ Clin Invest. 2011;121 (7):2750-2767. doi:10.1172/JCI45014

13. Lehmann BD, Pietenpol JA. Identification and use of biomarkers in treatment strategies for triple-negative breast cancer subtypes. J Pathol. 2014;232(2):142-150. doi:10.1002/path.4280

14. Mittendorf EA, Philips AV, Meric-Bernstam F, et al. PD-L1 expression in triple-negative breast cancer. Cancer Immunol Res. 2014;2 (4):361-370. doi:10.1158/2326-6066.CIR-13-0127 
15. Nanda R, Chow LQ, Dees EC, et al. Pembrolizumab in patients with advanced triple-negative breast cancer: phase Ib KEYNOTE-012 study. J Clin Oncol. 2016;34(21):2460-2467. doi:10.1200/JCO.2015.64.8931

16. Nanda R, Specht J, Dees C, et al. Abstract P6-10-03: KEYNOTE-012: long-lasting responses in a phase $\mathrm{Ib}$ study of pembrolizumab for metastatic triple-negative breast cancer (mTNBC). Cancer Res. 2017;77:P6-10.

17. Adams S, Schmid P, Rugo H, et al. Pembrolizumab monotherapy for previously treated metastatic triple-negative breast cancer: cohort A of the Phase 2 KEYNOTE-086 Study. Ann Oncol. 2018;30:397-404.

18. Cortés J, Lipatov O, Im S, et al. Keynote-119: phase 3 study of pembrolizumab (Pembro) versus single-agent chemotherapy (Chemo) for metastatic triple-negative breast cancer (Mtnbc). Ann Oncol. 2019;30(suppl_5):v851-v934. doi:10.1093/annonc/mdz394

19. Rugo HS, Delord JP, Im SA, et al. Safety and antitumor activity of pembrolizumab in patients with estrogen receptor-positive/human epidermal growth factor receptor 2-negative advanced breast cancer. Clin Cancer Res. 2018;24(12):2804-2811. doi:10.1158/1078-0432.CCR-173452

20. Dirix LY, Takacs I, Jerusalem G, et al. Avelumab, an anti-PD-L1 antibody, in patients with locally advanced or metastatic breast cancer: a phase 1b JAVELIN Solid Tumor study. Breast Cancer Res Treat. 2018;167(3):671-686. doi:10.1007/s10549-017-4537-5

21. Lee HT, Lee JY, Lim H, et al. Molecular mechanism of PD-1/PD-L1 blockade via anti-PD-L1 antibodies atezolizumab and durvalumab. Sci Rep. 2017;7(1):5532. doi:10.1038/s41598-017-06002-8

22. Emens LA, Cruz C, Eder JP, et al. Long-term clinical outcomes and biomarker analyses of atezolizumab therapy for patients with metastatic triple-negative breast cancer: a phase 1 study. JAMA Oncol. 2019;5(1):74-82. doi:10.1001/jamaoncol.2018.4224

23. Sansom DM, Walker LS. The role of CD28 and cytotoxic T-lymphocyte antigen-4 (CTLA-4) in regulatory T-cell biology. Immunol Rev. 2006;212:131-148. doi:10.1111/j.0105-2896.2006.00419.x

24. Rudd CE, Taylor A, Schneider H. CD28 and CTLA-4 coreceptor expression and signal transduction. Immunol Rev. 2009;229 (1):12-26. doi:10.1111/imr.2009.229.issue-1

25. Tarhini AA, Kirkwood JM. Tremelimumab (CP-675,206): a fully human anticytotoxic $\mathrm{T}$ lymphocyte-associated antigen 4 monoclonal antibody for treatment of patients with advanced cancers. Expert Opin Biol Ther. 2008;8(10):1583-1593. doi:10.1517/14712598. 8.10.1583

26. Naidoo J, Page DB, Wolchok JD. Immune checkpoint blockade. Hematol Oncol Clin North Am. 2014;28(3):585-600. doi:10.1016/j. hoc.2014.02.002

27. Vonderheide RH, LoRusso PM, Khalil M, et al. Tremelimumab in combination with exemestane in patients with advanced breast cancer and treatment-associated modulation of inducible costimulator expression on patient T cells. Clin Cancer Res. 2010;16(13):3485-3494. doi:10.1158/ 1078-0432.CCR-10-0505

28. McArthur HL, Diab A, Page DB, et al. A pilot study of preoperative single-dose ipilimumab and/or cryoablation in women with early-stage breast cancer with comprehensive immune profiling. Clin Cancer Res. 2016;22(23):5729-5737. doi:10.1158/1078-0432. CCR-16-0190

29. Sharabi AB, Nirschl CJ, Kochel CM, et al. Stereotactic radiation therapy augments antigen-specific PD-1-mediated antitumor immune responses via cross-presentation of tumor antigen. Cancer Immunol Res. 2015;3(4):345-355. doi:10.1158/2326-6066.CIR-14-0196

30. Pilones K, Emerson R, Formenti S, Robins H, Demaria S. Unique changes in the TCR repertoire of tumor-infiltrating lymphocytes underlie the synergy of radiotherapy with CTLA-4 blockade. J ImmunoTher Cancer. 2014;2(3):P141. doi:10.1186/2051-1426-2-S3-P141

31. Dovedi SJ, Adlard AL, Lipowska-Bhalla G, et al. Acquired resistance to fractionated radiotherapy can be overcome by concurrent PD-L1 blockade. Cancer Res. 2014;74(19):5458-5468. doi:10.1158/00085472.CAN-14-1258
32. Deng L, Liang $\mathrm{H}$, Burnette $\mathrm{B}$, et al. Irradiation and anti-PD-L1 treatment synergistically promote antitumor immunity in mice. $J$ Clin Invest. 2014;124(2):687-695. doi:10.1172/JCI67313

33. McArthur HL, Barker CA, Gucalp A, et al. A single-arm, phase II study assessing the efficacy of pembrolizumab (pembro) plus radiotherapy (RT) in metastatic triple negative breast cancer (mTNBC). J Clin Oncol. 2018;36(5_suppl):14. doi:10.1200/JCO.2018.36.5_suppl.14

34. Demaria S, Kawashima N, Yang AM, et al. Immune-mediated inhibition of metastases after treatment with local radiation and CTLA-4 blockade in a mouse model of breast cancer. Clin Cancer Res. 2005;11(2 Pt 1):728-734.

35. Jiang DM, Fyles A, Nguyen LT, et al. Phase I study of local radiation and tremelimumab in patients with inoperable locally recurrent or metastatic breast cancer. Oncotarget. 2019;10(31):2947-2958. doi:10.18632/oncotarget.26893

36. Zitvogel L, Apetoh L, Ghiringhelli F, Kroemer G. Immunological aspects of cancer chemotherapy. Nat Rev Immunol. 2008;8(1):59-73. doi: $10.1038 /$ nri2216

37. Adams S, Diamond JR, Hamilton E, et al. Atezolizumab plus nab-paclitaxel in the treatment of metastatic triple-negative breast cancer with 2-year survival follow-up: a phase 1b clinical trial. JAMA oncol. 2019;5(3):334-342. doi:10.1001/jamaoncol.2018.5152

38. Schmid P, Adams S, Rugo HS, et al. IMpassion130: updated overall survival (OS) from a global, randomized, double-blind, placebo-controlled, Phase III study of atezolizumab (atezo) + nab-paclitaxel (nP) in previously untreated locally advanced or metastatic triple-negative breast cancer (mTNBC). J Clin Oncol. 2019;37 (15_suppl): 1003

39. Schmid P, Adams S, Rugo HS, et al. Atezolizumab and nab-paclitaxel in advanced triple-negative breast cancer. $N$ Engl J Med. 2018;379 (22):2108-2121. doi:10.1056/NEJMoa1809615

40. Tolaney S, Savulsky C, Aktan G, et al. Abstract P5-15-02: phase 1b/2 study to evaluate eribulin mesylate in combination with pembrolizumab in patients with metastatic triple-negative breast cancer. Cancer Res. 2017;77(4 Supplement):P5-15-02.

41. Junttila TT, Li J, Johnston J, et al. Antitumor efficacy of a bispecific antibody that targets HER2 and activates T cells. Cancer Res. 2014;74(19):5561-5571. doi:10.1158/0008-5472.CAN-13-3622-T

42. Force J, Howie LJ, Abbott SE, et al. Early stage HER2-positive breast cancers not achieving a pCR from neoadjuvant trastuzumab- or pertuzumab-based regimens have an immunosuppressive phenotype. Clin Breast Cancer. 2018;18(5):410-417. doi:10.1016/j.clbc.2018.02.010

43. Muraro E, Comaro E, Talamini R, et al. Improved natural killer cell activity and retained anti-tumor $\operatorname{CD} 8(+) \mathrm{T}$ cell responses contribute to the induction of a pathological complete response in HER2-positive breast cancer patients undergoing neoadjuvant chemotherapy. J Transl Med. 2015;13:204. doi:10.1186/s12967-015-0567-0

44. Loi S, Giobbie-Hurder A, Gombos A, et al. Pembrolizumab plus trastuzumab in trastuzumab-resistant, advanced, HER2-positive breast cancer (PANACEA): a single-arm, multicentre, phase 1b-2 trial. Lancet Oncol. 2019;20(3):371-382. doi:10.1016/S1470-2045(18)30812-X

45. Emens L, Esteva F, Beresford M, et al. Abstract PD3-01: results from KATE2, a randomized phase 2 study of atezolizumab (atezo)+trastuzumab emtansine (T-DM1) vs placebo (pbo)+T-DM1 in previously treated HER2+ advanced breast cancer (BC). Cancer Res. 2019;79(4 Supplement):PD03-01.

46. Emens LA, Esteva FJ, Beresford M. Overall survival (Os) in Kate2, a phase 2 study of programmed death ligand 1 (Pd-L1) inhibitor atezolizumab (Atezo)+Trastuzumab Emtansine (T-Dm1) vs placebo (Pbo) $+\mathrm{T}-\mathrm{Dm} 1$ in previously treated Her2+ advanced breast cancer $(\mathrm{Bc})$. Ann Oncol. 2019;30(suppl_5):v104-v142. doi:10.1093/annonc/mdz242

47. Chia SKL, Bedard PL, Hilton J, et al. A phase I study of a PD-L1 antibody (Durvalumab) in combination with trastuzumab in HER-2 positive metastatic breast cancer (MBC) progressing on prior anti HER-2 therapies (CCTG IND.229)[NCT02649686]. J Clin Oncol. 2018;36(15_suppl):1029. doi:10.1200/JCO.2018.36.15_suppl.1029 
48. Jiao S, Xia W, Yamaguchi H, et al. PARP inhibitor upregulates PD-L1 expression and enhances cancer-associated immunosuppression. Clin Cancer Res. 2017;23(14):3711-3720. doi:10.1158/1078-0432.CCR$16-3215$

49. Domchek S, Postel-Vinay S, Im S-A, et al. 1191OPhase II study of olaparib (O) and durvalumab (D) (MEDIOLA): updated results in patients (pts) with germline BRCA-mutated (gBRCAm) metastatic breast cancer (MBC). Ann Oncol. 2019;30(Supplement_5):v477. doi:10.1093/annonc/mdz253.017

50. Konstantinopoulos PA, Waggoner SE, Vidal GA, et al. TOPACIO/ Keynote-162 (NCT02657889): a phase 1/2 study of niraparib + pembrolizumab in patients (pts) with advanced triple-negative breast cancer or recurrent ovarian cancer (ROC)-Results from ROC cohort. J Clin Oncol. 2018;36(15_suppl):106. doi:10.1200/JCO.2018. 36.15_suppl.106

51. Vinayak S, Tolaney SM, Schwartzberg L, et al. Open-label clinical trial of niraparib combined with pembrolizumab for treatment of advanced or metastatic triple-negative breast cancer. JAMA oncol. 2019;5(8):1132-1140. doi:10.1001/jamaoncol.2019.1029

52. Finn RS, Martin M, Rugo HS, et al. Palbociclib and letrozole in advanced breast cancer. N Engl J Med. 2016;375(20):1925-1936. doi:10.1056/NEJMoa1607303

53. Sledge GW Jr., Toi M, Neven P, et al. MONARCH 2: abemaciclib in combination with fulvestrant in women with HR+/HER2- advanced breast cancer who had progressed while receiving endocrine therapy. J Clin Oncol. 2017;35(25):2875-2884. doi:10.1200/JCO.2017.73. 7585
54. Slamon DJ, Neven P, Chia S, et al. Phase III randomized study of ribociclib and fulvestrant in hormone receptor-positive, human epidermal growth factor receptor 2-negative advanced breast cancer: MONALEESA-3. J Clin Oncol. 2018;36(24):2465-2472. doi:10.1200/ JCO.2018.78.9909

55. Goel S, DeCristo MJ, Watt AC, et al. CDK4/6 inhibition triggers anti-tumour immunity. Nature. 2017;548(7668):471-475. doi:10.1038/ nature 23465

56. Schaer DA, Beckmann RP, Dempsey JA, et al. The CDK4/6 inhibitor abemaciclib induces a $\mathrm{T}$ cell inflamed tumor microenvironment and enhances the efficacy of PD-L1 checkpoint blockade. Cell Rep. 2018;22(11):2978-2994. doi:10.1016/j.celrep.2018.02.053

57. Tolaney SM, Kabos P, Dickler MN, et al. Updated efficacy, safety, \& PD-L1 status of patients with HR+, HER2- metastatic breast cancer administered abemaciclib plus pembrolizumab. $J$ Clin Oncol. 2018;36(15_suppl):1059. doi:10.1200/JCO.2018.36.15_suppl.1059

58. Wolchok JD, Kluger H, Callahan MK, et al. Nivolumab plus ipilimumab in advanced melanoma. N Engl J Med. 2013;369(2):122-133. doi:10.1056/NEJMoa1302369

59. Larkin J, Chiarion-Sileni V, Gonzalez R, et al. Combined nivolumab and ipilimumab or monotherapy in untreated melanoma. $N$ Engl $J$ Med. 2015;373(1):23-34. doi:10.1056/NEJMoa1504030

60. Santa-Maria CA, Kato T, Park J-H, et al. A pilot study of durvalumab and tremelimumab and immunogenomic dynamics in metastatic breast cancer. Oncotarget. 2018;9(27):18985. doi:10.18632/oncotarget.v9i27

\section{Publish your work in this journal}

OncoTargets and Therapy is an international, peer-reviewed, open access journal focusing on the pathological basis of all cancers, potential targets for therapy and treatment protocols employed to improve the management of cancer patients. The journal also focuses on the impact of management programs and new therapeutic agents and protocols on patient perspectives such as quality of life, adherence and satisfaction. The manuscript management system is completely online and includes a very quick and fair peer-review system, which is all easy to use. Visit http://www.dovepress.com/ testimonials.php to read real quotes from published authors. 